\title{
Black-hole scattering with general spin directions from minimal-coupling amplitudes
}

\author{
Alfredo Guevara, ${ }^{1,2,3, *}$ Alexander Ochirov $\oplus^{4, \dagger}$ and Justin Vines $\oplus^{5, \$}$ \\ ${ }^{1}$ Perimeter Institute for Theoretical Physics, Waterloo, Ontario N2L 2Y5, Canada \\ ${ }^{2}$ Department of Physics \& Astronomy, University of Waterloo, Waterloo, Ontario N2L 3G1, Canada \\ ${ }^{3}$ CECs Valdivia \& Departamento de Física, Universidad de Concepción, \\ Casilla 160-C, Concepción, Chile \\ ${ }^{4}$ ETH Zürich, Institut für Theoretische Physik, Wolfgang-Pauli-Str. 27, 8093 Zürich, Switzerland \\ ${ }^{5}$ Max Planck Institute for Gravitational Physics (Albert Einstein Institute), \\ Am Mühlenberg 1, Potsdam 14476, Germany
}

(Received 17 September 2019; published 12 November 2019)

\begin{abstract}
We study the link between classical scattering of spinning black holes and quantum amplitudes for massive spin-s particles. Generic spin orientations of the black holes are considered, allowing their spins to be deflected on par with their momenta. We rederive the spin-exponentiated structure of the relevant treelevel amplitude from minimal coupling to Einstein's gravity, which in the $s \rightarrow \infty$ limit generates the black holes' complete series of spin-induced multipoles. The resulting scattering function is seen to encode in a simple way the known net changes in the black-hole momenta and spins at first post-Minkowskian order. We connect our findings to a rigorous framework developed elsewhere for computing such observables from amplitudes.
\end{abstract}

DOI: 10.1103/PhysRevD.100.104024

\section{INTRODUCTION}

Applying techniques from quantum field theory to the study of the classical two-body problem in general relativity (GR) has produced significant progress in the treatment of the post-Newtonian (PN) approximation [1-6], expanding in small speeds and weak fields. A particular focus of more recent interest has been the use of quantum scattering amplitudes to produce explicit classical results in the post-Minkowskian (PM) approximation [7,8], which resums the expansion in small speeds while still expanding in weak coupling. Several new contributions have been made to the understanding of conservative monopolar twobody dynamics up to 2PM order (through $\mathcal{O}\left(G^{2}\right)$, where $G$ is Newton's constant) using one-loop amplitudes [9-15], all ultimately confirming an equivalent classical solution from decades ago [16]. A significant milestone has been the first presentation of results at 3PM order from a two-loop amplitude calculation [17], using an arsenal of modern amplitude techniques [18-23].

\footnotetext{
*aguevara@pitp.ca

†aochirov@phys.ethz.ch

justin.vines@aei.mpg.de
}

Published by the American Physical Society under the terms of the Creative Commons Attribution 4.0 International license. Further distribution of this work must maintain attribution to the author(s) and the published article's title, journal citation, and DOI. Funded by SCOAP.
The first results for spin-orbit coupling in the PM scheme have been computed only recently, from purely classical considerations, at 1PM and 2PM orders [24,25]. Spin-orbit (or dipole) effects are universal in the two-body problem in GR, in that their form does not depend on the nature of the spinning bodies. It was shown in Refs. $[24,25]$ how they are determined by the parallel transport map along the geodesic worldline in the (regularized) spacetime metric sourced by monopolar bodies.

Going beyond the pole-dipole level, higher-multipole couplings specific to black holes (BHs) were treated at 1PM order in Ref. [26], by means of a classical effective action approach [4,5] matched to the linearized Kerr solution, to all orders in the spin-induced multipole expansion. In this paper we aim to fully reproduce the central results of Ref. [26] from amplitudes, exploiting and further substantiating the remarkable fact that the $\mathrm{BH}$ multipole structure up to the $2^{2 s}$-pole level is reproduced by considering spin- $s$ particles which are minimally coupled to gravity. This was first demonstrated in Ref. [27] up to the spin-2 level for the leading-PN-order corrections to the two-body interaction potential, following work along similar lines in Refs. [28,29].

A generalization of minimal-coupling amplitudes to arbitrary spins $s$ has been proposed recently in Ref. [30] using a new massive spinor-helicity formalism. Through the course of Refs. [10,31-33], there has emerged a consistent picture of how these amplitudes encode the 




FIG. 1. Three-point amplitude.

complete tower of spinning-BH multipole moments, at least at 1PM order, when one lets the spin quantum number $s$ tend to infinity. ${ }^{1}$ Concerning explicit specifications of the classical two-body dynamics, the final results of Ref. [32] allowed arbitrary spin orientations but restricted to leading orders in the PN expansion, while those of Ref. [31] were complete at 1PM order but restricted to the case where the $\mathrm{BH}$ spin vectors are aligned with the orbital angular momentum. In this aligned-spin case, the two-body scattering is confined to a single constant orbital plane, and the spin vectors are conserved, pointing orthogonally to that plane. In the general case, both the orbital plane and the spin vectors are rotated in the course of the interaction.

In this paper we consider general spin directions at full 1PM order. The staring point is the tree-level amplitude for one-graviton exchange between two massive spin-s particles shown in Fig. 2. We compute its holomorphic classical limit (HCL) [10] by gluing two of the minimalcoupling three-point amplitudes [30] depicted in Fig. 1. We streamline the treatment of the spin-exponentiated structure of such amplitudes, which was the focus of Ref. [31], by incorporating additional Lorentz boosts [33] into the spin exponentials. Finally, we adapt to our needs a general formalism [34,35] for extracting gauge-invariant classical observables from amplitudes. It has already been used in Ref. [35] to compute the net changes in the momenta and spins for two-body scattering at 1PM order, reproducing the results for BHs up to quadrupolar order from minimally coupled spin-1 particles. Here we extend such calculation to arbitrary spins $s$, and in the limit $s \rightarrow \infty$ obtain all orders in the BH multipole expansions at 1PM order [26].

\section{MINIMAL COUPLING TO GRAVITY}

In this section we review the angular-momentum exponentiation that is inherent to the gravitational coupling of spinning black holes and the corresponding amplitudes.

\footnotetext{
${ }^{1}$ We note that Refs. [10,31,32] have also treated spin contributions at 2PM order, and that Ref. [33] has also considered radiative effects via a classical double copy with spin.
}

At the linearized-gravity level, the classical stress-energy tensor serving as an effective source for a single Kerr black hole with mass $m$, classical momentum $p^{\mu}=m u^{\mu}$ and spin $S^{\mu}=m a^{\mu}$ is [26]

$T_{\mathrm{BH}}^{\mu \nu}(x)=\frac{1}{m} \int d \tau p^{(\mu} \exp (a * \partial)^{\nu)}{ }_{\rho} p^{\rho} \delta^{(4)}(x-u \tau)$,

where we have used the shorthand notation

$$
(a * b)^{\mu \nu}=\epsilon^{\mu \nu \alpha \beta} a_{\alpha} b_{\beta} .
$$

The spin transversality condition $p \cdot a=0$ is also assumed. The corresponding coupling of the $\mathrm{BH}$ to gravity is

$$
\begin{aligned}
S_{\mathrm{BH}} & =-\frac{\kappa}{2} \int \hat{d}^{4} k h_{\mu \nu}(k) T_{\mathrm{BH}}^{\mu \nu}(-k) \\
& =-\kappa \int \hat{d}^{4} k \hat{\delta}(2 p \cdot k) p^{(\mu} \exp (a * i k)^{\nu}{ }_{\rho} p^{\rho} h_{\mu \nu}(k),
\end{aligned}
$$

where the coupling constant is $\kappa=\sqrt{32 \pi G}$. Here and below the hats over the delta functions and measures encode appropriate positive or negative powers of $2 \pi$, respectively. Putting the graviton on shell, $h_{\mu \nu}(k) \rightarrow \hat{\delta}\left(k^{2}\right) \varepsilon_{\mu} \varepsilon_{\nu}$, we can rewrite the characteristic angular-momentum exponential in another form

$h_{\mu \nu}(k) T_{\mathrm{BH}}^{\mu \nu}(-k)=\hat{\delta}\left(k^{2}\right) \hat{\delta}(p \cdot k)(p \cdot \varepsilon)^{2} \exp \left(-i \frac{k_{\mu} \varepsilon_{\nu} S^{\mu \nu}}{p \cdot \varepsilon}\right)$,

now involving a transverse spin tensor $S^{\mu \nu}$

$$
S^{\mu \nu}=\epsilon^{\mu \nu \rho \sigma} p_{\rho} a_{\sigma} \Rightarrow p_{\mu} S^{\mu \nu}=0 .
$$

More explicitly, the above transition relies on the equality

$$
(p \cdot \varepsilon)^{j-1} \varepsilon_{\mu}\left[(a * i k)^{j}\right]^{\mu}{ }_{\nu} p^{\nu}=\left(-i k_{\mu} \varepsilon_{\nu} S^{\mu \nu}\right)^{j},
$$

that is easiest verified in the frame and gauge where $k=\left(k^{0}, 0,0, k^{0}\right), \varepsilon=\left(0, \varepsilon^{1}, \pm i \varepsilon^{1}, 0\right)$ and $p=\left(p^{0}, p^{1}, 0, p^{0}\right)$.

In Ref. [31] we discovered that the same exponential is hidden inside the minimal-coupling amplitudes proposed recently by Arkani-Hamed, Huang and Huang [30]

$$
\begin{aligned}
& \mathcal{M}_{3}\left(p_{1}^{\{a\}},-p_{2}^{\{b\}}, k^{+}\right)=-\frac{\kappa}{2} \frac{\left\langle 1^{a} 2^{b}\right\rangle^{\odot 2 s}}{m^{2 s-2}} x^{2}, \\
& \mathcal{M}_{3}\left(p_{1}^{\{a\}},-p_{2}^{\{b\}}, k^{-}\right)=-\frac{\kappa}{2} \frac{\left[1^{a} 2^{b}\right]^{\odot 2 s}}{m^{2 s-2}} x^{-2} .
\end{aligned}
$$

The arguments of scattering amplitudes are treated as incoming, so the present choice corresponds to the momentum configuration shown in Fig. 1. Here and below the symbol $\odot$ denotes tensor product symmetrized over each 
massive particle's $2 s$ little-group indices $\left\{a_{1}, \ldots, a_{2 s}\right\}$ and $\left\{b_{1}, \ldots, b_{2 s}\right\}$. Furthermore, $x$ is the positive-helicity factor

$x=\frac{\left[k\left|p_{1}\right| r\right\rangle}{m\langle k r\rangle}=-\frac{\sqrt{2}}{m}\left(p_{1} \cdot \varepsilon^{+}\right)=\left[\frac{\sqrt{2}}{m}\left(p_{1} \cdot \varepsilon^{-}\right)\right]^{-1}$,

that is dimensionless and independent of the reference momentum $r$ on the on-shell three-point kinematics [30].

Now one can start by noticing that the amplitudes $\mathcal{M}_{3}^{(0)}=-\kappa\left(p_{1} \cdot \varepsilon^{ \pm}\right)^{2}$, given by the scalar case of Eq. (7), correspond precisely to the $S^{\mu \nu}=0$ case of the vertex (4). Moreover, we were able to recast the spin structure of the amplitudes (7) in an exponential form [31]

$$
\begin{aligned}
& \left\langle 2^{b} 1^{a}\right\rangle^{\odot 2 s}=\left[\left.2^{b}\right|^{\odot 2 s} \exp \left(-i \frac{k_{\mu} \varepsilon_{\nu}^{+} \bar{\sigma}^{\mu \nu}}{p_{1} \cdot \varepsilon^{+}}\right) \mid 1^{a}\right]^{\odot 2 s}, \\
& {\left[2^{b} 1^{a}\right]^{\odot 2 s}=\left\langle\left. 2^{b}\right|^{\odot 2 s} \exp \left(-i \frac{k_{\mu} \varepsilon_{\nu}^{-} \sigma^{\mu \nu}}{p_{1} \cdot \varepsilon^{-}}\right) \mid 1^{a}\right\rangle^{\odot 2 s},}
\end{aligned}
$$

featuring a tensor-product version of the chiral and antichiral spinorial generators

$\sigma^{\mu \nu}=\frac{i}{4}\left[\sigma^{\mu} \bar{\sigma}^{\nu}-\sigma^{\nu} \bar{\sigma}^{\mu}\right], \quad \bar{\sigma}^{\mu \nu}=\frac{i}{4}\left[\bar{\sigma}^{\mu} \sigma^{\nu}-\bar{\sigma}^{\nu} \sigma^{\mu}\right]$.

In Ref. [31] we could translate between the spin-operator exponentials (9) and the classical-spin exponential (4) and thus identify the minimal-coupling amplitudes (7) with Kerr black holes. (A complementary identification was done in Ref. [32] by matching to the Wilson coefficients in the one-body effective field theory of a Kerr black hole $[4,6]$.) Such a translation involved sticking to either chiral or antichiral representation, so that one of the amplitudes in Eq. (7) contains no apparent dependence on the spin operator. The correct symmetric dependence on the classical spins was restored in Ref. [31] via a notion of generalized expectation value (GEV), which involved division by the product of the polarization tensors of the incoming and outgoing avatars of the $\mathrm{BH}$. In this paper we follow an alternative path: we recover the entire spin information from the amplitudes by combining the spinor-helicity formalism with the covariant approach to multipoles introduced in Ref. [33], while the GEV will only serve to fix the normalization.

\section{GENERAL INTEGER-SPIN SETUP}

Although the minimal-coupling amplitudes II of Ref. [30] are valid for both integer and half-integer spins, for simplicity we will concentrate on the former case. Spin$s$ polarization tensors are constructed as $[31,32]$

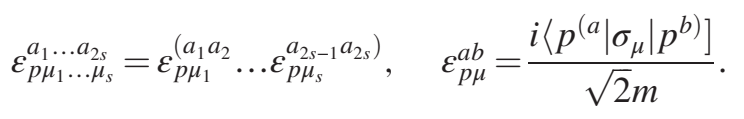

We adopt the spinor-helicity conventions of Ref. [36], so the spin-1 polarization vectors are spacelike and obey the standard properties that are expected from them:

$$
\begin{gathered}
p \cdot \varepsilon_{p}^{a b}=0, \\
\varepsilon_{p \mu}^{a b} \varepsilon_{p \nu a b}=-\eta_{\mu \nu}+\frac{p_{\mu} p_{\nu}}{m^{2}}, \\
\varepsilon_{p 11} \cdot \varepsilon_{p}^{11}=\varepsilon_{p 22} \cdot \varepsilon_{p}^{22}=2 \varepsilon_{p 12} \cdot \varepsilon_{p}^{12}=-1, \\
\left(\varepsilon_{p \mu}^{a b}\right)^{*}=\varepsilon_{p \mu a b}=\epsilon_{a c} \epsilon_{b d} \varepsilon_{p \mu}^{c d} .
\end{gathered}
$$

In particular, the last line follows from the conjugation rule

$\left(\lambda_{p \alpha}{ }^{a}\right)^{*}=\operatorname{sgn}\left(p^{0}\right) \tilde{\lambda}_{p \dot{\alpha} a} \Leftrightarrow\left(\tilde{\lambda}_{p \dot{\alpha}}{ }^{a}\right)^{*}=-\operatorname{sgn}\left(p^{0}\right) \lambda_{p \alpha a}$,

which implements the fact that in the little group SU(2) upper and lower indices are related by complex conjugation.

Since the polarization tensors are essentially symmetrized tensor products $\varepsilon_{p}^{\odot s}$, the action of the Lorentz generators is trivially induced by the vector representation

$$
\Sigma^{\mu \nu, \sigma}{ }_{\tau}=i\left[\eta^{\mu \sigma} \delta_{\tau}^{\nu}-\eta^{\nu \sigma} \delta_{\tau}^{\mu}\right]
$$

namely,

$$
\begin{aligned}
\left(\Sigma^{\mu \nu}\right)^{\sigma_{1} \ldots \sigma_{s}}{ }_{\tau_{1} \ldots \tau_{s}}= & \Sigma^{\mu \nu, \sigma_{1}}{ }_{\tau_{1}} \delta_{\tau_{2}}^{\sigma_{2}} \ldots \delta_{\tau_{s}}^{\sigma_{s}} \\
& +\cdots+\delta_{\tau_{1}}^{\sigma_{1}} \ldots \delta_{\tau_{s-1}}^{\sigma_{s-1}} \Sigma^{\mu \nu, \sigma_{s}} \tau_{\tau_{s}} .
\end{aligned}
$$

These matrices realize the Lorentz algebra on the oneparticle states of spin $s$, which are represented by the polarization tensors (11).

A more convenient spin quantity to deal with is the PauliLubanski vector

$$
S_{\lambda}=\frac{1}{2 m} \epsilon_{\lambda \mu \nu \rho} S^{\mu \nu} p^{\rho} .
$$

Here $S^{\mu \nu}$ is the spin tensor, the transverse part of which can be reconstructed from the vector as

$$
S_{\perp}^{\mu \nu}=\frac{1}{m} \epsilon^{\mu \nu \rho \sigma} p_{\rho} S_{\sigma} \Rightarrow p_{\mu} S_{\perp}^{\mu \nu}=0 .
$$

Understanding Eq. (16) in the operator sense, we can derive the general form of one-particle matrix element of the Pauli-Lubanski spin operator, here denoted by $\Sigma^{\mu}$,

$$
\begin{aligned}
\varepsilon_{p}^{\{a\}} \cdot \Sigma^{\mu} \cdot \varepsilon_{p}^{\{b\}}= & \frac{s(-1)^{s-1}}{2 m}\left\{\left\langle p^{\left(a_{1}\right.}\left|\sigma^{\mu}\right| p^{\left(b_{1}\right.}\right]\right. \\
& \left.+\left[p^{\left(a_{1}\right.}\left|\bar{\sigma}^{\mu}\right| p^{\left(b_{1}\right.}\right\rangle\right\} \epsilon^{a_{2} b_{2}} \ldots \epsilon^{\left.\left.a_{2 s}\right) b_{2 s}\right)} .
\end{aligned}
$$

One way to give meaning to this formula is to lower one set of indices and set it equal to the other: it then produces an expectation value 


$$
\frac{\varepsilon_{p\{a\}} \cdot \Sigma^{\mu} \cdot \varepsilon_{p}^{\{a\}}}{\varepsilon_{p\{a\}} \cdot \varepsilon_{p}^{\{a\}}}= \begin{cases}s s_{p}^{\mu}, & a_{1}=\ldots=a_{2 s}=1, \\ (s-1) s_{p}^{\mu}, & \sum_{j=1}^{2 s} a_{j}=2 s+1, \\ (s-2) s_{p}^{\mu}, & \sum_{j=1}^{2 s} a_{j}=2 s+2, \\ \cdots & \\ -s s_{p}^{\mu}, & a_{1}=\ldots=a_{2 s}=2,\end{cases}
$$

where we have also accounted for the nontrivial normalization of the tensors. This shows that spin is quantized in terms of the unit-spin vector

$$
s_{p}^{\mu}=-\frac{1}{2 m}\left\{\left\langle p_{1}\left|\sigma^{\mu}\right| p^{1}\right]+\left[p_{1}\left|\bar{\sigma}^{\mu}\right| p^{1}\right\rangle\right\}
$$

that is transverse and spacelike, $p \cdot s_{p}=0, s_{p}^{2}=-1$. This vector is familiar from textbook discussions of the Dirac spin, in which context it may be written as $\frac{1}{2 m} \bar{u}_{p 1} \gamma^{\mu} \gamma^{5} u_{p}^{1}=-\frac{1}{2 m} \bar{u}_{p 2} \gamma^{\mu} \gamma^{5} u_{p}^{2}$. According to Eq. (19), this vector corresponds to the spin quantization axis and identifies the $(2 s+1)$ distinct wave functions $\varepsilon_{p}^{1 \ldots 12 \ldots 2}$ with states of definite spin projection.

Moving on toward the scattering context, let us consider a three-point kinematics $p_{1}+k=p_{2}$ shown in Fig. 1. A naive extension of the spin matrix element (18), now between states with different momenta of mass $m$, is

$$
\begin{aligned}
\varepsilon_{2}^{\{b\}} \cdot \Sigma^{\mu}\left[p_{\mathrm{a}}\right] \cdot \varepsilon_{1}^{\{a\}}= & \frac{s}{4 m^{2 s}}\left\{\left\langle 1^{a}\left|\sigma^{\mu}\right| 2^{b}\right]+\left[1^{a}\left|\bar{\sigma}^{\mu}\right| 2^{b}\right\rangle\right\} \\
& \odot\left\{\left\langle 1^{a} 2^{b}\right\rangle-\left[1^{a} 2^{b}\right]\right\} \odot\left\langle 1^{a} 2^{b}\right\rangle \odot(s-1) \\
& \odot\left[1^{a} 2^{b}\right]^{\odot(s-1)} .
\end{aligned}
$$

Here we have encoded the symmetrization of the littlegroup indices into the modified tensor-product symbol $\odot$, and the indices on the right-hand side should be regarded as abstract placeholders. It is important to stress that the symmetrization encoded in the symbol $\odot$ only acts inside the two $\mathrm{SU}(2)$-index sets $\{a\}$ and $\{b\}$ separately, as symmetrizing a little-group index of momentum $p_{1}$ with that of $p_{2}$ would be mathematically inconsistent.

Notice that in Eq. (21) we must specify that the PauliLubanski operator is defined with respect to the average momentum $p_{\mathrm{a}}=\left(p_{1}+p_{2}\right) / 2$. It is this momentum that we will associate with the classical momentum $p_{\mathrm{a}}^{\mu}=m u_{\mathrm{a}}^{\mu}$ of one of the incoming black holes, so it makes sense to define a spin vector to be orthogonal to it. Equation (21) treats the chiral and antichiral spinors on an equal footing and generalizes the spin-1 matrix element considered in Ref. [31]. However, even in the spin-1 case the angularmomentum exponentiation (9), present in the exclusively chiral and antichiral spinorial representations, was found to be opaque at the level of such a matrix element. The reason for that is physically important. As discussed in Ref. [4], a consistent picture of spin-induced multipoles of a point-like particle must be formulated in the particle's rest frame, in which the spin does not precess [37]. Therefore, the formula (21) is too naive, as it involves a spin operator defined for momentum $p_{\mathrm{a}}$ but acts with it on the states with momenta $p_{\mathrm{a}} \pm k / 2$. The cure for that is to take into account additional Lorentz boosts, which we will now proceed to do.

\section{ANGULAR-MOMENTUM EXPONENTIATION}

Bautista and one of the current authors have recently argued that all spin multipoles of the amplitude can be extracted through a finite Lorentz boost [33]. This boost is needed to bridge the gap between two states with different momenta. In this way, the quantum picture is made consistent with the classical notion of spin-induced multipoles of a pointlike object on a worldline [4]. Here we introduce such a construction in terms of the spinor-helicity variables. Its equivalence to the covariant formalism of Ref. [33] is explained in the Appendix.

To start, we note that any two four-vectors $p_{1}$ and $p_{2}$ of equal mass $m$ may be related by

$$
p_{2}^{\rho}=\exp \left(i \mu_{12} p_{1}^{\mu} p_{2}^{\nu} \Sigma_{\mu \nu}\right)_{\sigma}^{\rho} p_{1}^{\sigma},
$$

where we have used the generators (14). The numeric prefactor in the exponent is explicitly

$$
\mu_{12}=\frac{\log \left[\frac{1}{m^{2}}\left(p_{1} \cdot p_{2}+\sqrt{\left(p_{1} \cdot p_{2}\right)^{2}-m^{2}}\right)\right]}{\sqrt{\left(p_{1} \cdot p_{2}\right)^{2}-m^{2}}}=\frac{1}{m^{2}}+\mathcal{O}\left(k^{2}\right) .
$$

Here we are only interested in the strictly on-shell setup, for which $k^{2}=\left(p_{2}-p_{1}\right)^{2}=0$. The corresponding spinorial transformations are

$$
\begin{aligned}
\left|2^{b}\right\rangle & =U_{12}{ }_{a}^{b} \exp \left(\frac{i}{m^{2}} p_{1}^{\mu} k^{\nu} \sigma_{\mu \nu}\right)\left|1^{a}\right\rangle, \\
\left.\mid 2^{b}\right] & \left.=U_{12^{b}{ }_{a}} \exp \left(\frac{i}{m^{2}} p_{1}^{\mu} k^{\nu} \bar{\sigma}_{\mu \nu}\right) \mid 1^{a}\right],
\end{aligned}
$$

where $U_{12} \in \mathrm{SU}(2)$ is a little-group transformation that depends on the specifics of the massive-spinor realization. The duality properties of the spinorial generators (10) allow us to easily rewrite the above exponents as

$$
\frac{i}{m^{2}} p_{1}^{\mu} k^{\nu} \sigma_{\mu \nu, \alpha}^{\beta}=k \cdot a_{\alpha}^{\beta}, \quad \frac{i}{m^{2}} p_{1}^{\mu} k^{\nu} \bar{\sigma}_{\mu \nu, \dot{\beta}}{ }_{\dot{\beta}}=-k \cdot a_{\dot{\beta}}^{\dot{\alpha}},
$$

where we have defined chiral representations for the PauliLubanski operators 


$$
\begin{aligned}
& a_{\alpha}^{\mu, \beta}=\frac{1}{2 m^{2}} \epsilon^{\mu \nu \rho \sigma} p_{\mathrm{a} \nu} \sigma_{\rho \sigma, \alpha}{ }^{\beta}, \\
& a_{\dot{\beta}}^{\mu, \dot{\alpha}}=\frac{1}{2 m^{2}} \epsilon^{\mu \nu \rho \sigma} p_{\mathrm{a} \nu} \bar{\sigma}_{\rho \sigma, \dot{\alpha}}{ }_{\dot{\beta}} .
\end{aligned}
$$

(Note that the product $k \cdot a$ is insensitive to the difference between $p_{1}$ and $p_{\mathrm{a}}=p_{1}+k / 2$ in the above definitions, so we could pick the latter for further convenience.) Extension to the higher-spin states represented by tensor products of massive spinors is analogous to Eq. (15), e.g.,

$$
\begin{aligned}
\left(a^{\mu}\right)_{\alpha_{1} \ldots \alpha_{2 s}}^{\beta_{1} \ldots \beta_{2 s}}= & a^{\mu,}{ }_{\alpha_{1}} \beta_{1} \delta_{\alpha_{2}}^{\beta_{2}} \ldots \delta_{\alpha_{2 s}}^{\beta_{2 s}} \\
& +\cdots+\delta_{\alpha_{1}}^{\beta_{1}} \ldots \delta_{\alpha_{2 s-1}}^{\beta_{2 s-1}} a^{\mu,}{ }_{2 s}{ }_{2 s},
\end{aligned}
$$

so we have

$$
\begin{aligned}
& \left.\left.|2\rangle^{\odot 2 s}=e^{k \cdot a}\left\{U_{12}|1\rangle\right\}^{\odot 2 s}, \quad \mid 2\right]^{\odot 2 s}=e^{-k \cdot a}\left\{U_{12} \mid 1\right]\right\}^{\odot 2 s}, \\
& \left\langle\left. 2\right|^{\odot 2 s}=\left\{U_{12}\langle 1|\right\}^{\odot 2 s} e^{-k \cdot a}, \quad\left[\left.2\right|^{\odot 2 s}=\left\{U_{12}[1 \mid\}^{\odot 2 s} e^{k \cdot a},\right.\right.\right.
\end{aligned}
$$

where the second line follows from the antisymmetry of $\sigma^{\mu \nu}$ and $\bar{\sigma}^{\mu \nu}$ in the sense of $\epsilon^{\alpha \beta} \sigma^{\mu \nu,}{ }_{\beta}^{\gamma} \epsilon_{\gamma \delta}=-\sigma^{\mu \nu,}{ }_{\delta}{ }^{2}$.

Let us now inspect the spin dependence of the threepoint amplitudes. In Ref. [31] we used their representation (9) for that. In terms of the same Pauli-Lubanski operators (26), they can be rewritten in a simpler form:

$$
\begin{aligned}
& \mathcal{M}_{3}^{(s)}\left(k^{+}\right)=-\frac{\kappa x^{2}}{2 m^{2 s-2}}\left[\left.2\right|^{\odot 2 s} e^{-2 k \cdot a} \mid 1\right]^{\odot 2 s}, \\
& \mathcal{M}_{3}^{(s)}\left(k^{-}\right)=-\frac{\kappa x^{-2}}{2 m^{2 s-2}}\left\langle\left. 2\right|^{\odot 2 s} e^{2 k \cdot a} \mid 1\right\rangle^{\odot 2 s},
\end{aligned}
$$

which can be derived from Eq. (7) using the identities

$$
\begin{gathered}
{\left[1^{a} k\right]=x\left\langle 1^{a} k\right\rangle, \quad\left[2^{b} k\right]=x\left\langle 2^{b} k\right\rangle,} \\
{\left[1^{a} 2^{b}\right]=-\left\langle 1^{a} 2^{b}\right\rangle+\frac{x}{m}\left\langle 1^{a} k\right\rangle\left\langle k 2^{b}\right\rangle .}
\end{gathered}
$$

The apparent spin dependence in the amplitude formulas above is of the form $e^{\mp 2 k \cdot a}$, whereas there seems to be no such dependence in the original formulas (7) from Ref. [30]. This apparent contradiction is resolved by taking into account the transformations (24): the true angularmomentum dependence inherent to the minimal-coupling amplitudes is independent of the spinorial basis. [Indeed, it must also match the covariant formula (A3).] For example, the plus-helicity amplitude (7a) involves $\langle 12\rangle^{\odot 2 s}$, which in the chiral representation is simply

$$
\langle 21\rangle^{\odot 2 s}=\left\{U_{12}\langle 1|\right\}^{\odot 2 s} e^{-k \cdot a}|1\rangle^{\odot 2 s},
$$

whereas in the antichiral representation it is

$$
\left[\left.2\right|^{\odot 2 s} e^{-2 k \cdot a} \mid 1\right]^{\odot 2 s}=\left\{U_{12}[1 \mid\}^{\odot 2 s} e^{-k \cdot a} \mid 1\right]^{\odot 2 s} .
$$

As pointed out in the Appendix, it is now natural to strip the spin-states to cleanly obtain the spin dependence. Alternatively, in the classical (and arbitrary-spin) limit we should treat the operator in-between as a C-number. In that case, both expressions above become unambiguously

$$
\lim _{s \rightarrow \infty} m^{2 s}\left(U_{12}\right)^{\odot 2 s} e^{-k \cdot a} .
$$

The factor of $m^{2 s}$ cancels in the actual amplitudes:

$$
\begin{aligned}
& \mathcal{M}_{3}^{(\infty)}\left(k^{+}\right) \approx-\frac{\kappa}{2} m^{2} x^{2} e^{-k \cdot a} \lim _{s \rightarrow \infty}\left(U_{12}\right)^{\odot 2 s}, \\
& \mathcal{M}_{3}^{(\infty)}\left(k^{-}\right) \approx-\frac{\kappa}{2} m^{2} x^{-2} e^{k \cdot a} \lim _{s \rightarrow \infty}\left(U_{12}\right)^{\odot 2 s} .
\end{aligned}
$$

The remaining unitary factor of $\left(U_{12}\right)^{\odot 2 s}$ parametrizes an arbitrary little-group transformation that corresponds to the choice of the spin quantization axis (20). As such, it is inherently quantum-mechanical and therefore should be removed in the classical limit. Indeed, it also appears in the simple product of polarization tensors

$$
\begin{aligned}
\lim _{s \rightarrow \infty} \varepsilon_{2} \cdot \varepsilon_{1}= & \lim _{s \rightarrow \infty} \frac{1}{m^{2 s}}\langle 21\rangle^{\odot s} \odot[21]^{\odot s} \\
= & \lim _{s \rightarrow \infty} \frac{1}{m^{2 s}}\left\{U_{12}\langle 1|\right\}^{\odot s} e^{-k \cdot a}|1\rangle^{\odot s} \\
& \times\left\{U_{12}[1 \mid\}^{\odot s} e^{k \cdot a} \mid 1\right]^{\odot s}=\lim _{s \rightarrow \infty}\left(U_{12}\right)^{\odot 2 s},
\end{aligned}
$$

where $a$ is defined by Eq. (27) but with half as many slots. So we interpret the factor of $\left(U_{12}\right)^{\odot 2 s}$ as the state normalization in accord with the notion of GEV of Ref. [31].

\section{FOUR-POINT AMPLITUDE}

We are now ready to compute the four-point amplitude that contains the complete information about classical 1PM scattering of two spinning black holes, with masses $m_{\mathrm{a}}$ and $m_{\mathrm{b}}$. In the rigorous framework of Kosower, Maybee and O'Connell [34] for computing the momentum deflection in the spinless case, the tree-level contribution to the impulse expectation value is

$$
\begin{aligned}
\Delta p_{\mathrm{a}}^{\mu}= & \lim _{\hbar \rightarrow 0} \int \hat{d}^{4} p_{1} \hat{\delta}_{+}\left(p_{1}^{2}-m_{\mathrm{a}}^{2}\right) \int \hat{d}^{4} p_{2} \hat{\delta}_{+}\left(p_{2}^{2}-m_{\mathrm{a}}^{2}\right) \\
& \times \int \hat{d}^{4} p_{3} \hat{\delta}_{+}\left(p_{3}^{2}-m_{\mathrm{b}}^{2}\right) \int \hat{d}^{4} p_{4} \hat{\delta}_{+}\left(p_{4}^{2}-m_{\mathrm{b}}^{2}\right) \\
& \times \hat{\delta}\left(p_{1}+p_{3}-p_{2}-p_{4}\right) \psi_{\mathrm{a}}\left(p_{1}\right) \psi_{\mathrm{a}}^{*}\left(p_{2}\right) \psi_{\mathrm{b}}\left(p_{3}\right) \psi_{\mathrm{b}}^{*}\left(p_{4}\right) \\
& \times k^{\mu} e^{-i k \cdot b / \hbar} i \mathcal{M}_{4}\left(p_{1},-p_{2}, p_{3},-p_{4}\right),
\end{aligned}
$$

where $k=p_{2}-p_{1}$ and the wave functions $\psi_{\mathrm{a}, \mathrm{b}}$ describe quantum-mechanical wave packets with momenta well 


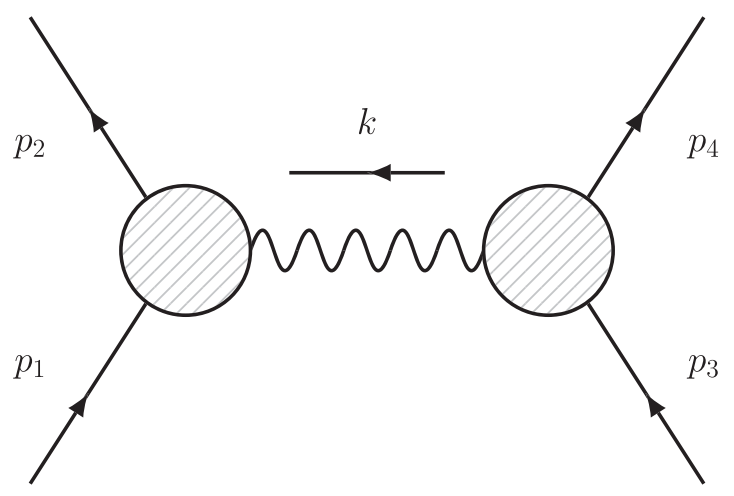

FIG. 2. Four-point amplitude for elastic scattering of two distinct massive particles.

approximated by the classical momenta $p_{\mathrm{a}, \mathrm{b}}$. It then leads to a schematic formula

$\Delta p_{\mathrm{a}}^{\mu}=\|\left\langle\int \hat{d}^{4} k \hat{\delta}\left(2 p_{\mathrm{a}} \cdot k\right) \hat{\delta}\left(2 p_{\mathrm{b}} \cdot k\right) k^{\mu} e^{-i k \cdot b / \hbar} i \mathcal{M}_{4}(k) \|\right.$.

Here the angle-bracket notation of Ref. [34] involves a careful analysis of suitable wave functions $\psi_{\mathrm{a}, \mathrm{b}}$ and powers of $\hbar$, and roughly amounts to setting the momenta to their classical values as follows

$k^{\mu}=\hbar \bar{k}^{\mu} \rightarrow 0, \quad p_{1}^{\mu}, p_{2}^{\mu} \rightarrow m_{\mathrm{a}} u_{\mathrm{a}}^{\mu}, \quad p_{3}^{\mu}, p_{4}^{\mu} \rightarrow m_{\mathrm{b}} u_{\mathrm{b}}^{\mu}$.

First of all, we note that in the quantum-mechanical setting of Ref. [34] both $p_{1}$ and $p_{2}$ are associated with the momentum of the first incoming black hole. This is consistent with the equitable identification

$$
p_{\mathrm{a}}=\left(p_{1}+p_{2}\right) / 2, \quad p_{\mathrm{b}}=\left(p_{3}+p_{4}\right) / 2,
$$

that we will follow. Moreover, the classical limit (38) prescribes inspecting soft-graviton exchange in the $t=k^{2}$ channel, in which the graviton's momentum is taken to zero uniformly. Here, however, we are going to adhere to an alternative strategy of the HCL [10]: we compute the residue of the scattering amplitude on the pole at $t=0$ on finite complex kinematics and analytically continue the result to real kinematics at a later stage. As shown in Fig. 2, the four-point amplitude then conveniently factorizes into two three-point ones:

$$
\begin{aligned}
& \mathcal{M}_{4}^{\left(s_{\mathrm{a}}, s_{\mathrm{b}}\right)}\left(p_{1},-p_{2}, p_{3},-p_{4}\right) \\
& =\frac{-1}{t} \sum_{ \pm} \mathcal{M}_{3}^{\left(s_{\mathrm{a}}\right)}\left(p_{1},-p_{2}, k^{ \pm}\right) \mathcal{M}_{3}^{\left(s_{\mathrm{b}}\right)}\left(p_{3},-p_{4},-k^{\mp}\right)+\mathcal{O}\left(t^{0}\right) \\
& =\frac{-(\kappa / 2)^{2}}{m_{\mathrm{a}}^{2 s_{\mathrm{a}}-2} m_{\mathrm{b}}^{2 s_{\mathrm{b}}-2} t}\left(x_{\mathrm{a}}^{2} x_{\mathrm{b}}^{-2}\langle 21\rangle^{\odot 2 s_{\mathrm{a}}}[43]^{\odot 2 s_{\mathrm{b}}}\right. \\
& \left.+x_{\mathrm{a}}^{-2} x_{\mathrm{b}}^{2}[21]^{\odot 2 s_{\mathrm{a}}}\langle 43\rangle^{\odot 2 s_{\mathrm{b}}}\right)+\mathcal{O}\left(t^{0}\right),
\end{aligned}
$$

of which we now have complete understanding.
The helicity factors enter the above amplitude in simple combinations evaluated on the pole kinematics as

$$
x_{\mathrm{a}} / x_{\mathrm{b}}=\gamma(1-v), \quad x_{\mathrm{b}} / x_{\mathrm{a}}=\gamma(1+v),
$$

where we have introduced the following interchangeable variables that describe the total energy of the black-hole scattering process:

$$
\gamma=\frac{1}{\sqrt{1-v^{2}}}=\frac{p_{\mathrm{a}} \cdot p_{\mathrm{b}}}{m_{\mathrm{a}} m_{\mathrm{b}}}=u_{\mathrm{a}} \cdot u_{\mathrm{b}}
$$

Evaluating the spin-dependent terms using Eq. (28) and taking into account the direction of $k^{\mu}$, we get

$$
\begin{aligned}
\mathcal{M}_{4}^{\left(s_{\mathrm{a}}, s_{\mathrm{b}}\right)}= & \frac{-(\kappa / 2)^{2} \gamma^{2}}{m_{\mathrm{a}}^{2 s_{\mathrm{a}}-2} m_{\mathrm{b}}^{2 s_{\mathrm{b}}-2} t} \\
& \times\left((1-v)^{2}\left\{U_{12}\langle 1|\right\}^{\odot 2 s_{\mathrm{a}}} \exp \left(-k \cdot a_{\mathrm{a}}\right)|1\rangle^{\odot 2 s_{\mathrm{a}}}\right. \\
& \times\left\{U_{34}[3 \mid\}^{\odot 2 s_{\mathrm{b}}} \exp \left(-k \cdot a_{\mathrm{b}}\right) \mid 3\right]^{\odot 2 s_{\mathrm{b}}} \\
& +(1+v)^{2}\left\{U_{12}[1 \mid\}^{\odot 2 s_{\mathrm{a}}} \exp \left(k \cdot a_{\mathrm{a}}\right) \mid 1\right]^{\odot 2 s_{\mathrm{a}}} \\
& \left.\times\left\{U_{34}\langle 3|\right\}^{\odot 2 s_{\mathrm{b}}} \exp \left(k \cdot a_{\mathrm{b}}\right)|3\rangle^{\odot 2 s_{\mathrm{b}}}\right)+\mathcal{O}\left(t^{0}\right) .
\end{aligned}
$$

It is straightforward to check that the same result is obtained if we choose to Lorentz-transform the states symmetrically to their averages: $p_{1}, p_{2} \rightarrow p_{\mathrm{a}}$ and $p_{3}, p_{4} \rightarrow p_{\mathrm{b}}$.

Before we take the classical limit, we should note that the above contractions of the Pauli-Lubanski pseudovector are parity-odd. To obtain a parity even expression, we observe that on the pole kinematics $k^{2}=0$ the Levi-Civita spin contractions satisfy

$$
\begin{aligned}
& i \epsilon_{\mu \nu \rho \sigma} p_{\mathrm{a}}^{\mu} p_{\mathrm{b}}^{\nu} k^{\rho} a_{\mathrm{a}}^{\sigma}=m_{\mathrm{a}} m_{\mathrm{b}} \gamma v\left(k \cdot a_{\mathrm{a}}\right), \\
& i \epsilon_{\mu \nu \rho \sigma} p_{\mathrm{a}}^{\mu} p_{\mathrm{b}}^{\nu} k^{\rho} a_{\mathrm{b}}^{\sigma}=m_{\mathrm{a}} m_{\mathrm{b}} \gamma v\left(k \cdot a_{\mathrm{b}}\right) .
\end{aligned}
$$

These equalities can be derived by squaring the lefthand sides and computing the resulting Gram determinants using that $k^{2}=p_{\mathrm{a}} \cdot k=p_{\mathrm{b}} \cdot k=p_{\mathrm{a}} \cdot a_{\mathrm{a}}=p_{\mathrm{b}} \cdot a_{\mathrm{b}}=0$. Therefore, introducing a two-form constructed from two initial $\mathrm{BH}$ momenta

$$
w^{\mu \nu}=\frac{2 p_{\mathrm{a}}^{[\mu} p_{\mathrm{b}}^{\nu]}}{m_{\mathrm{a}} m_{\mathrm{b}} \gamma v}, \quad[w * a]_{\mu}=\frac{1}{2} \epsilon_{\mu \nu \alpha \beta} w^{\alpha \beta} a^{\nu},
$$

and stripping the unitary transition factors $U_{12}^{\odot 2 s_{\mathrm{a}}}$ and $U_{34}^{\odot 2 s_{\mathrm{b}}}$ via the GEV, we obtain the classical limit of the scattering amplitude (43) as

$$
\left\langle\mathcal{M}_{4}(k)\right\rangle=-\left(\frac{\kappa}{2}\right)^{2} \frac{m_{\mathrm{a}}^{2} m_{\mathrm{b}}^{2}}{k^{2}} \gamma^{2} \sum_{ \pm}(1 \pm v)^{2} \exp \left[ \pm i\left(k \cdot w * a_{0}\right)\right]
$$




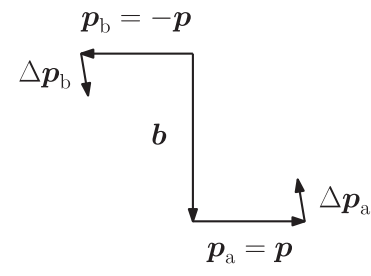

FIG. 3. The BH three-momenta in the center-of-mass frame and the impact parameter between them.

where $a_{0}^{\mu}=a_{\mathrm{a}}^{\mu}+a_{\mathrm{b}}^{\mu}$ is the total spin pseudovector. Note that from now on we consider the above expression to be valid for any values of transfer momentum momentum $k$.

As suggested by Eq. (37) and the scattering-angle formula of Ref. [12], in the classical picture we consider the transfer momentum $k$ as a Fourier variable dual to the impact parameter $b$, which is a spacelike vector orthogonal to both of initial momenta, $b \cdot p_{\mathrm{a}}=b \cdot p_{\mathrm{b}}=0$. Therefore, we define the scattering function

$$
\left\langle\mathcal{M}_{4}(b)\right\rangle=\int \hat{d}^{4} k \hat{\delta}\left(2 p_{\mathrm{a}} \cdot k\right) \hat{\delta}\left(2 p_{\mathrm{b}} \cdot k\right) e^{-i k \cdot b}\left\langle\mathcal{M}_{4}(k)\right\rangle
$$

The above Fourier transform is easiest performed in the center-of-mass $(\mathrm{COM})$ frame, where $p_{\mathrm{a}}=\left(E_{\mathrm{a}}, \boldsymbol{p}\right)$ and $p_{\mathrm{b}}=\left(E_{\mathrm{b}},-\boldsymbol{p}\right)$, see Fig. 3. In this frame the eikonal integration measure [12] becomes explicitly

$$
\begin{aligned}
& \int \hat{d}^{4} k \hat{\delta}\left(2 p_{\mathrm{a}} \cdot k\right) \hat{\delta}\left(2 p_{\mathrm{b}} \cdot k\right) e^{-i k \cdot b} \\
& \left.\stackrel{\text { COM frame }}{=} \frac{1}{4\left(E_{\mathrm{a}}+E_{\mathrm{b}}\right)|\boldsymbol{p}|} \int \hat{d}^{2} \boldsymbol{k} e^{i \boldsymbol{k} \cdot \boldsymbol{b}}\right|_{k^{0}=\boldsymbol{p} \cdot \boldsymbol{k}=0}
\end{aligned}
$$

In other words, the integration is strictly spacelike and restricted by $\boldsymbol{p} \cdot \boldsymbol{k}=0$ to the two-dimensional subspace orthogonal to the initial momenta, which is the same subspace where the impact parameter is defined. Using $\left(E_{\mathrm{a}}+E_{\mathrm{b}}\right)|\boldsymbol{p}|=m_{\mathrm{a}} m_{\mathrm{b}} \gamma v$, we compute

$$
\begin{aligned}
\left\langle\mathcal{M}_{4}(\boldsymbol{b})\right\rangle= & \frac{\kappa^{2} m_{\mathrm{a}} m_{\mathrm{b}} \gamma}{16 v} \sum_{ \pm}(1 \pm v)^{2} \\
& \times\left.\int \frac{d^{2} \boldsymbol{k}}{(2 \pi)^{2} \boldsymbol{k}^{2}} \exp \left[i \boldsymbol{k} \cdot\left(\boldsymbol{b} \mp\left[w * a_{0}\right]_{2 d}\right)\right]\right|_{p \cdot \boldsymbol{k}=0} \\
= & -\frac{\kappa^{2} m_{\mathrm{a}} m_{\mathrm{b}} \gamma}{32 \pi v} \sum_{ \pm}(1 \pm v)^{2} \log \left|\boldsymbol{b} \mp\left[w * a_{0}\right]_{2 d}\right|
\end{aligned}
$$

Here by $\left[w * a_{0}\right]_{2 d}$ we have denoted the appropriate spacelike projection of the four-vector $w * a_{0}$. However, recall that

$$
\left[w * a_{0}\right]^{\mu}=\frac{\epsilon^{\mu \nu \rho \sigma} a_{0 \nu} p_{\mathrm{a} \rho} p_{\mathrm{b} \sigma}}{m_{\mathrm{a}} m_{\mathrm{b}} \gamma v}
$$

i.e., the vector $w * a_{0}$ is transverse to $p_{\mathrm{a}}$ and $p_{\mathrm{b}}$ and hence lies in the same plane as $\boldsymbol{k}$ and $\boldsymbol{b}$. Therefore, no information is lost in the two-dimensional projection above, so we can safely uplift the scattering function (49) to its Lorentzinvariant form

$$
\left\langle\mathcal{M}_{4}(b)\right\rangle=-G m_{\mathrm{a}} m_{\mathrm{b}} \frac{\gamma}{v} \sum_{ \pm}(1 \pm v)^{2} \log \sqrt{-\left(b \mp w * a_{0}\right)^{2}}
$$

\section{LINEAR AND ANGULAR IMPULSES}

In this section we relate the scattering function (51) in the impact-parameter space to the classical changes in linear and angular momentum of a $\mathrm{BH}$ after gravitational scattering off another $\mathrm{BH}$. This problem was treated to all orders in spins at 1PM order by one of the present authors [26], producing the result (rewritten in the mostly-minus metric convention)

$$
\Delta p_{\mathrm{a}}^{\mu}=G m_{\mathrm{a}} m_{\mathrm{b}} \Re Z^{\mu},
$$

$\Delta a_{\mathrm{a}}^{\mu}=-\frac{G m_{\mathrm{b}}}{m_{\mathrm{a}}}\left[p_{\mathrm{a}}^{\mu}\left(a_{\mathrm{a}} \cdot \Re Z\right)+\epsilon^{\mu \nu \rho \sigma}\left(\Im Z_{\nu}\right) p_{\mathrm{a} \rho} a_{\mathrm{a} \sigma}\right]$,

in terms of an auxiliary complex vector

$Z^{\mu}=\frac{\gamma}{v} \sum_{ \pm}(1 \pm v)^{2}\left[\eta^{\mu \nu} \mp i(* w)^{\mu \nu}\right] \frac{\left(b \mp w * a_{0}\right)_{\nu}}{\left(b \mp w * a_{0}\right)^{2}}$

Now we can observe that differentiating the scattering function (51) automatically produces its real and imaginary parts:

$$
\begin{gathered}
\frac{\partial}{\partial b^{\mu}}\left\langle\mathcal{M}_{4}(b)\right\rangle=-G m_{\mathrm{a}} m_{\mathrm{b}} \Re Z_{\mu}, \\
\frac{\partial}{\partial a^{\mu}}\left\langle\mathcal{M}_{4}(b)\right\rangle=G m_{\mathrm{a}} m_{\mathrm{b}} \Im Z_{\mu} .
\end{gathered}
$$

Using the known solution (52), we can identify

$$
\begin{aligned}
\Delta p_{\mathrm{a}}^{\mu} & =-\frac{\partial}{\partial b_{\mu}}\left\langle\mathcal{M}_{4}(b)\right\rangle, \\
\Delta a_{\mathrm{a}}^{\mu} & =\frac{1}{m_{\mathrm{a}}^{2}}\left[p_{\mathrm{a}}^{\mu} a_{\mathrm{a}}^{\nu} \frac{\partial}{\partial b^{\nu}}-\epsilon^{\mu \nu \rho \sigma} p_{\mathrm{a} \nu} a_{\mathrm{a} \rho} \frac{\partial}{\partial a_{\mathrm{a}}^{\sigma}}\right]\left\langle\mathcal{M}_{4}(b)\right\rangle .
\end{aligned}
$$

At this point, we have merely matched the derivatives of our scattering function (51) to the known result (52). Let us now promote this empirical matching to a derivation, under the assumption that our approach is consistent with that of Ref. [34]. 
Indeed, the first line of Eq. (55) gives precisely the impulse formula (37) from Ref. [34]. So let us focus on the second line. In the concurrent preprint [35], Maybee, O'Connell and one of the current authors have extended the classical-limit approach of Ref. [34] to include corrections in spin. Their starting point for (the expectation value of) the lowest-order angular impulse is

$$
\begin{aligned}
\Delta S_{\mathrm{a}}^{\mu}= & \| \iint \hat{d}^{4} k \hat{\delta}\left(2 p_{\mathrm{a}} \cdot k\right) \hat{\delta}\left(2 p_{\mathrm{b}} \cdot k\right) e^{-i k \cdot b} \\
& \times\left(-\frac{i}{m_{\mathrm{a}}^{2}} p_{\mathrm{a}}^{\mu} S_{\mathrm{a}}^{\nu} k_{\nu} \mathcal{M}_{4}(k)+\left[S_{\mathrm{a}}^{\mu}, i \mathcal{M}_{4}(k)\right]\right) \| .
\end{aligned}
$$

Here the amplitude is considered to be a function of a oneparticle spin vector acting on the space of physical spin degrees of freedom, i.e., the little-group indices. Therefore, we interpret it as the matrix element

$$
\left(S_{\mathrm{a}}^{\mu}\right)_{\{a\}}\{b\}=(-1)^{s} \varepsilon_{\mathrm{a}\left\{a_{1} \ldots a_{2 s}\right\}} \cdot \Sigma^{\mu} \cdot \varepsilon_{\mathrm{a}}^{\left\{b_{1} \ldots b_{2 s}\right\}},
$$

where the prefactor of $(-1)^{s}$ is due to the spacelike normalization of the polarization vectors in Eq. (12c). The explicit form for such a spin vector at finite spin is given in Eq. (18). It corresponds to the generator of the little-group transformations: as an operator it satisfies the so (3) algebra in the rest frame of $p_{\mathrm{a}}$. This can also be stated covariantly as

$$
\left[S_{\mathrm{a}}^{\mu}, S_{\mathrm{a}}^{\nu}\right]=\frac{i}{m_{\mathrm{a}}} \epsilon^{\mu \nu \rho \sigma} p_{\mathrm{a} \rho} S_{\mathrm{a} \sigma} .
$$

As $\mathcal{M}_{4}$ is a function of $S_{\mathrm{a}}^{\mu}$, these so(3) rotations act as

$$
\left[S_{\mathrm{a}}^{\mu}, \mathcal{M}_{4}\right]=\frac{i}{m_{\mathrm{a}}} \epsilon^{\mu \nu \rho \sigma} p_{\mathrm{a} \nu} S_{\mathrm{a} \rho} \frac{\partial \mathcal{M}_{4}}{\partial S_{\mathrm{a}}^{\sigma}} .
$$

Therefore, we obtain the formula for the change in rescaled spin

$$
\begin{aligned}
\Delta a_{\mathrm{a}}^{\mu}= & \frac{1}{m_{\mathrm{a}}^{2}}\left\langle\left\langle\int \hat{d}^{4} k \hat{\delta}\left(2 p_{\mathrm{a}} \cdot k\right) \hat{\delta}\left(2 p_{\mathrm{b}} \cdot k\right) e^{-i k \cdot b}\right.\right. \\
& \times\left[p_{\mathrm{a}}^{\mu} a_{\mathrm{a}}^{\nu}\left(-i k_{\nu}\right)-\epsilon^{\mu \nu \rho \sigma} p_{\nu} a_{\mathrm{a} \rho} \frac{\partial}{\partial a_{\mathrm{a}}^{\sigma}}\right] \mathcal{M}_{4}(k) \|,
\end{aligned}
$$

which maps directly to the second line of Eq. (55). Now that the impulse formulas (55) have meaning on their own, we see that plugging the scattering function (51) gives a novel derivation for the complete 1PM solution (52).

\section{SUMMARY AND DISCUSSION}

In this paper we have obtained the dynamically complete solution to the (net) problem of conservative spinning black-hole scattering at 1PM order as given in Ref. [26], using minimal-coupling scattering amplitudes with arbitrarily large quantum spin [30]. We have rederived the spin-exponentiated structure of such three-point amplitudes in four dimensions in a way that takes into account the Lorentz boost between the incoming and outgoing momenta [33]. In Ref. [31] this boost was overlooked but effectively restored by the introduction of the generalized expectation value. Here we have shown that considering this boost streamlines the discussion of the spin exponentiation, as well as allows for a cleaner connection to the classical notion of spin in general relativity [4].

We have computed the change of the momentum and spin of the scattered black holes at 1PM order using a fourpoint one-graviton-exchange amplitude, which in the holomorphic classical limit [10] is factorized into two threepoint minimal-coupling amplitudes. We have adopted the formulas of Ref. $[34,35]$ in a way that allowed us to extract the full spin dependence of the linear and angular impulses of the black holes. In this way, we obtained a complete match to the known solution 1PM solution [26], which allows for spins of the incoming black holes in arbitrary directions. This is also a significant step forward from the simpler case of the angular momenta aligned perpendicular to the scattering plane considered in Ref. [31]. It is promising that our calculation displayed a sufficiently uniform level of complexity all the way between the starting point and the final result, even despite the more complex nature of the quantum degrees of freedom. This is thanks to the remarkable fact that the spin multipoles of a black hole exponentiate [26,38], which we could exploit and thus avoid explicit multipole expansions.

There are several interesting future directions. One of the most relevant ones is the extension to higher loop, or PM, orders $[9,12,13,15]$, which may require to include radiative corrections [33-35] and finite-size effects [39-42]. One could also attempt to consider higher curvature corrections, such as the ones in Refs. [43,44]. It is also interesting to explore the test-body limit $[26,45]$ to improve our understanding of the effective potential in the sense of Ref. [46]. Furthermore, it may prove beneficial to use the double-copy approach to quantum gravity amplitudes [22,23,47,48], which has recently seen classical extensions [49-51], and in this way apply it to the binary-inspiral problem [33,5257]. Additional insight may come from studying the same problem in a supersymmetric setting [58].

In conclusion, our work, together with Refs. [32,35], opens the way to higher-order calculations for the spin effects in classical black-hole scattering using the modern amplitude techniques in an on shell and gauge-invariant framework.

\section{ACKNOWLEDGMENTS}

We thank Fabián Bautista, David Kosower, Ben Maybee and Donal O'Connell for illuminating discussions and collaboration on related topics. A. G. acknowledges support via Conicyt Grant No. 21151647. A. O. has received 
funding from the European Union's Horizon 2020 research and innovation programme under the Marie SkłodowskaCurie Grant Agreement No. 746138. Research at Perimeter Institute is supported in part by the Government of Canada through the Department of Innovation, Science and Economic Development Canada and by the Province of Ontario through the Ministry of Economic Development, Job Creation and Trade.

\section{APPENDIX: SPIN MULTIPOLES FROM BOOSTS}

Here we review the construction of Ref. [33] applied to the three-point amplitudes and show how it simplifies in the spinor-helicity formalism. Consider the three-point amplitude in the covariant form as given there:

$$
\mathcal{M}_{3}^{(s)}=\mathcal{M}_{3}^{(0)} \varepsilon_{2} \cdot \exp \left(-i \frac{k_{\mu} \varepsilon_{\nu} \Sigma^{\mu \nu}}{p_{1} \cdot \varepsilon}\right) \cdot \varepsilon_{1},
$$

where $\varepsilon_{1}$ and $\varepsilon_{2}$ are spin- $s$ polarization tensors, the generators $\Sigma^{\mu \nu}$ are given in Eq. (15), and $\mathcal{M}_{3}^{(0)}=-\kappa\left(p_{1}\right.$. $\varepsilon)^{2}$ corresponds to the gravitational interaction of a scalar particle. It was proposed that in order to extract classical multipoles (forming representations of the little group in the sense of Ref. [4]) the spin states must be evaluated at the same momenta. On the three-point kinematics, the polarization states for $p_{1}$ and $p_{2}$ are related via

$$
\varepsilon_{2}=\exp \left(\frac{i}{m^{2}} p_{1}^{\mu} k^{\nu} \Sigma_{\mu \nu}\right) \tilde{\varepsilon}_{1}, \quad \tilde{\varepsilon}_{1}=U_{12}^{(s)} \varepsilon_{1},
$$

where $U_{12}^{(s)}$ is a tensor representation of an $\mathrm{SO}(3)$ littlegroup transformation. Note that in the rest frame of particle 1 the Lorentz transformation $p_{1}^{\mu} k^{\nu} \Sigma_{\mu \nu}=m k^{i} \Sigma^{0 i}$ is nothing but the canonical choice for the boost needed to shift $p_{1}$ to $p_{1}+k$. One can show that the two exponents commute on the three-point kinematics, so

$$
\begin{aligned}
\mathcal{M}_{3}^{(s)} & =\mathcal{M}_{3}^{(0)} \tilde{\varepsilon}_{1} \exp \left(-\frac{i}{m^{2}} p_{1}^{\mu} k^{\nu} \Sigma_{\mu \nu}\right) \exp \left(-i \frac{k_{\mu} \varepsilon_{\nu} \Sigma^{\mu \nu}}{p_{1} \cdot \varepsilon}\right) \varepsilon_{1} \\
& =\mathcal{M}_{3}^{(0)} \tilde{\varepsilon}_{1} \exp \left(-i \frac{k_{\mu} \varepsilon_{\nu} \Sigma_{\perp}^{\mu \nu}}{p_{1} \cdot \varepsilon}\right) \varepsilon_{1},
\end{aligned}
$$

where we have defined

$$
\Sigma_{\perp}^{\mu \nu}=\Sigma^{\mu \nu}+\frac{2}{m^{2}} p_{1}^{[\mu} \Sigma^{\nu] \rho} p_{1 \rho}, \quad p_{1 \mu} \Sigma_{\perp}^{\mu \nu}=0,
$$

as the operator that corresponds to the transverse spin tensor (17). Being a transverse tensor, it can be used to construct representations of the little group. The first such $j$ spin multipoles of e.g., Ref. [4] are recovered by expanding the exponential to order $j$ and stripping $\varepsilon_{1}$ and $\tilde{\varepsilon}_{1}$. For finite spin $s$, it was observed that this exponential truncates at order $2 s$, whereas Eq. (A1) truncates at order $s$ [33].
Let us now apply the spinor-helicity formalism to the above argument. Picking for concreteness the negative helicity, it was shown in [33] that Eq. (A1) can be rewritten as our amplitude:

$$
\mathcal{M}_{3}^{(s)}=\frac{\mathcal{M}_{3}^{(0)}}{m^{2 s}}\left\langle\left. 2\right|^{\odot 2 s} \exp \left(-i \frac{k_{\mu} \varepsilon_{\nu}^{-} \sigma^{\mu \nu}}{p_{1} \cdot \varepsilon^{-}}\right) \mid 1\right\rangle^{\odot 2 s} .
$$

On the other hand, in Ref. [31] we noted that in the chiral spinor-variable basis self-duality of $\sigma^{\mu \nu}$ implies

$$
-i \frac{k_{\mu} \varepsilon_{\nu}^{-} \sigma^{\mu \nu}}{p_{1} \cdot \varepsilon^{-}}=-2 i \frac{k_{\mu} \varepsilon_{\nu}^{-} \sigma_{\perp}^{\mu \nu}}{p_{1} \cdot \varepsilon^{-}}=2 k \cdot a,
$$

where $a^{\mu}$ is given by (27) and $\sigma_{\perp}^{\mu \nu}$ is the transverse projection of $\sigma^{\mu \nu}$, as in Eq. (A4). The crucial factor of two arises here because in the spinor variables we cannot distinguish between the orbital or intrinsic pieces of the angular momentum. Indeed, the $p_{1} \rightarrow p_{2}$ boost considered in Eq. (A2) acts on the chiral basis as

$$
\frac{i}{m^{2}} p_{1}^{\mu} k^{\nu} \sigma_{\mu \nu}=k \cdot a \Rightarrow|2\rangle^{\odot 2 s}=e^{k \cdot a}\left\{U_{12}|1\rangle\right\}^{\odot 2 s}
$$

in accord with Eqs. (25) and (28) in the main text. This boost compensates the factor of two in Eq. (A6), so

$$
\begin{aligned}
\mathcal{M}_{3}^{(s)} & =\frac{\mathcal{M}_{3}^{(0)}}{m^{2 s}}\left\{U_{12}\langle 1|\right\}^{\odot 2 s} e^{-k \cdot a} e^{2 k \cdot a}|1\rangle^{\odot 2 s} \\
& =\frac{\mathcal{M}_{3}^{(0)}}{m^{2 s}}\left\{U_{12}\langle 1|\right\}^{\odot 2 s} e^{k \cdot a}|1\rangle^{\odot 2 s} .
\end{aligned}
$$

Now compare this to Eq. (A3), where two distinct exponentials combined into an exponential of a $\mathrm{SO}(3)$ rotation (A4). We see that in the four-dimensional chiral spinor basis it trivialized down to two exponentials, identical up to a numerical prefactor.

One might see an apparent contradiction in Eq. (A7). Namely, that the right-hand side involves the little-group rotation $k \cdot a$ preserving $p_{1}$, whereas the left-hand side corresponds to a boost $p_{1} \rightarrow p_{1}+k$. The reason that this is consistent is because Eq. (A7) is a chirality-dependent statement. In fact, the corresponding relation for the antichiral spinors involves a sign flip:

$$
\frac{i}{m^{2}} p_{1}^{\mu} k^{\nu} \bar{\sigma}_{\mu \nu}=-k \cdot a,
$$

as given in Eq. (25). More concretely, consider the following relations

$$
\begin{aligned}
{\left[e^{k \cdot a}\right]_{\alpha}^{\beta} p_{1 \beta \dot{\beta}}\left[e^{-k \cdot a}\right]_{\dot{\alpha}}^{\dot{\beta}} } & =p_{1 \alpha \dot{\alpha}}, \\
{\left[e^{k \cdot a}\right]_{\alpha}^{\beta} p_{1 \beta \dot{\beta}}\left[e^{k \cdot a}\right]_{\dot{\alpha}}^{\dot{\beta}} } & =p_{2 \alpha \dot{\alpha}},
\end{aligned}
$$


where $p_{i \alpha \dot{\alpha}}=\left|i^{a}\right\rangle_{\alpha}\left[\left.i_{a}\right|_{\dot{\alpha}}\right.$ as usual. The first relation is simply the statement that the Pauli-Lubanski operator generates little-group rotations, whereas the second relation shows that thanks to the sign flip $k$. $a$ can effectively act as a boost. Therefore, Eq. (A7) and Eq. (A9) contain no real contradiction and reflect the "square root" nature of the spinor-helicity representation.
[1] B. R. Holstein and J. F. Donoghue, Phys. Rev. Lett. 93, 201602 (2004).

[2] W. D. Goldberger and I. Z. Rothstein, Phys. Rev. D 73, 104029 (2006).

[3] R. A. Porto, Phys. Rev. D 73, 104031 (2006).

[4] M. Levi and J. Steinhoff, J. High Energy Phys. 09 (2015) 219.

[5] R. A. Porto, Phys. Rep. 633, 1 (2016).

[6] M. Levi, arXiv:1807.01699.

[7] D. Neill and I. Z. Rothstein, Nucl. Phys. B877, 177 (2013).

[8] T. Damour, Phys. Rev. D 94, 104015 (2016).

[9] F. Cachazo and A. Guevara, arXiv:1705.10262.

[10] A. Guevara, J. High Energy Phys. 04 (2019) 033.

[11] T. Damour, Phys. Rev. D 97, 044038 (2018).

[12] N. E. J. Bjerrum-Bohr, P. H. Damgaard, G. Festuccia, L. Plante, and P. Vanhove, Phys. Rev. Lett. 121, 171601 (2018).

[13] C. Cheung, I. Z. Rothstein, and M. P. Solon, Phys. Rev. Lett. 121, 251101 (2018).

[14] A. Koemans Collado, P. Di Vecchia, and R. Russo, Phys. Rev. D 100, 066028 (2019).

[15] A. Cristofoli, N. E. J. Bjerrum-Bohr, P. H. Damgaard, and P. Vanhove, Phys. Rev. D 100, 084040 (2019).

[16] K. Westpfahl, Fortschr. Phys. 33, 417 (1985).

[17] Z. Bern, C. Cheung, R. Roiban, C.-H. Shen, M. P. Solon, and M. Zeng, Phys. Rev. Lett. 122, 201603 (2019).

[18] Z. Bern, L. J. Dixon, D. C. Dunbar, and D. A. Kosower, Nucl. Phys. B425, 217 (1994).

[19] Z. Bern, L. J. Dixon, D. C. Dunbar, and D. A. Kosower, Nucl. Phys. B435, 59 (1995).

[20] R. Britto, F. Cachazo, and B. Feng, Nucl. Phys. B725, 275 (2005).

[21] H. Kawai, D. Lewellen, and S. Tye, Nucl. Phys. B269, 1 (1986).

[22] Z. Bern, J. J. M. Carrasco, and H. Johansson, Phys. Rev. D 78, 085011 (2008).

[23] H. Johansson and A. Ochirov, J. High Energy Phys. 11 (2015) 046.

[24] D. Bini and T. Damour, Phys. Rev. D 96, 104038 (2017).

[25] D. Bini and T. Damour, Phys. Rev. D 98, 044036 (2018).

[26] J. Vines, Classical Quantum Gravity 35, 084002 (2018).

[27] V. Vaidya, Phys. Rev. D 91, 024017 (2015).

[28] B. R. Holstein and A. Ross, arXiv:0802.0715.

[29] B. R. Holstein and A. Ross, arXiv:0802.0716.

[30] N. Arkani-Hamed, T.-C. Huang, and Y.-t. Huang, arXiv: 1709.04891.

[31] A. Guevara, A. Ochirov, and J. Vines, J. High Energy Phys. 09 (2019) 056.
[32] M.-Z. Chung, Y.-T. Huang, J.-W. Kim, and S. Lee, J. High Energy Phys. 04 (2019) 156.

[33] Y. F. Bautista and A. Guevara, arXiv:1903.12419.

[34] D. A. Kosower, B. Maybee, and D. O'Connell, J. High Energy Phys. 02 (2019) 137.

[35] B. Maybee, D. O'Connell, and J. Vines, arXiv:1906.09260.

[36] A. Ochirov, J. High Energy Phys. 04 (2018) 089.

[37] S. Weinberg, Gravitation and Cosmology: Principles and Applications of the General Theory of Relativity (John Wiley and Sons, New York, 1972).

[38] R. O. Hansen, J. Math. Phys. (N.Y.) 15, 46 (1974).

[39] D. Bini, T. Damour, and G. Faye, Phys. Rev. D 85, 124034 (2012).

[40] M. Levi and J. Steinhoff, J. High Energy Phys. 06 (2015) 059.

[41] B. Chen, G. Compère, Y. Liu, J. Long, and X. Zhang, arXiv: 1901.05370.

[42] S. Cai and K.-D. Wang, arXiv:1906.06850 [Phys. Rev. D (to be published)].

[43] A. Brandhuber and G. Travaglini, arXiv:1905.05657.

[44] W. T. Emond and N. Moynihan, arXiv:1905.08213.

[45] J. Vines, J. Steinhoff, and A. Buonanno, Phys. Rev. D 99, 064054 (2019).

[46] A. Antonelli, A. Buonanno, J. Steinhoff, M. van de Meent, and J. Vines, Phys. Rev. D 99, 104004 (2019).

[47] Z. Bern, J. J. M. Carrasco, and H. Johansson, Phys. Rev. Lett. 105, 061602 (2010).

[48] H. Johansson and A. Ochirov, J. High Energy Phys. 01 (2016) 170.

[49] R. Monteiro, D. O'Connell, and C. D. White, J. High Energy Phys. 12 (2014) 056.

[50] W. D. Goldberger and A. K. Ridgway, Phys. Rev. D 95, 125010 (2017).

[51] A. Luna, R. Monteiro, I. Nicholson, A. Ochirov, D. O'Connell, N. Westerberg, and C. D. White, J. High Energy Phys. 04 (2017) 069.

[52] W. D. Goldberger, J. Li, and S. G. Prabhu, Phys. Rev. D 97, 105018 (2018).

[53] W. D. Goldberger and A. K. Ridgway, Phys. Rev. D 97, 085019 (2018).

[54] J. Li and S. G. Prabhu, Phys. Rev. D 97, 105019 (2018).

[55] C.-H. Shen, J. High Energy Phys. 11 (2018) 162.

[56] J. Plefka, J. Steinhoff, and W. Wormsbecher, Phys. Rev. D 99, 024021 (2019).

[57] J. Plefka, C. Shi, J. Steinhoff, and T. Wang, Phys. Rev. D 100, 086006 (2019).

[58] S. Caron-Huot and Z. Zahraee, J. High Energy Phys. 07 (2019) 179. 